\title{
Circular RNA circ_0001006 aggravates cardiac hypertrophy via miR- 214-3p/PAK6 axis
}

\author{
Xuefeng Lin ${ }^{1,2,3,4,5,6}$, Liqin Zhang ${ }^{6}$, Wei Zhang ${ }^{7}$, Xinjun Lei ${ }^{1}$, Qun Lu' ${ }^{1}$, Aiqun Ma ${ }^{1,2,3,4}$ \\ ${ }^{1}$ Department of Cardiovascular Medicine, The First Affiliated Hospital of Xi' an Jiaotong University, Xi' an, Shanxi \\ 710061, PR China \\ ${ }^{2}$ Key Laboratory of Molecular Cardiology, Xi'an Jiaotong University, Xi'an, Shanxi 710061, PR China \\ ${ }^{3}$ Institute of Cardiovascular Channelopathy, Xi'an Jiaotong University, Xi'an, Shanxi 7100161, PR China \\ ${ }^{4}$ Key Laboratory of Environment and Genes Related to Diseases, Xi'an Jiaotong University, Ministry of Education, \\ Xi'an, Shanxi 7100161, PR China \\ ${ }^{5}$ Department of Cardiovascular Medicine, The First Affiliated Hospital of Baotou Medical College, Baotou, Inner \\ Mongolia 014010, PR China \\ ${ }^{6}$ Baotou Medical College, Baotou, Inner Mongolia 014010, PR China \\ ${ }^{7}$ ECG Room, Xi'an Children's Hospital, Xi'an, Shanxi 710061, PR China
}

Correspondence to: Aiqun Ma; email: mabakefen242954@163.com, https://orcid.org/0000-0003-1629-0412

Keywords: circular RNAs, cardiac hypertrophy, miRNA, gene expression, heart disease

Received: April 1, $2021 \quad$ Accepted: July 30, 2021

Published: March 6, 2022

Copyright: (C) 2021 Lin et al. This is an open access article distributed under the terms of the Creative Commons Attribution License (CC BY 3.0), which permits unrestricted use, distribution, and reproduction in any medium, provided the original author and source are credited.

\section{ABSTRACT}

Aim: Circular RNAs (circRNAs) control gene expression in a series of physiological and pathological processes, but their role in heart disease is unknown. This research illustrates the role and potential mechanism of circRNA in cardiac hypertrophy.

Methods and Results: In this report, we found that circular RNA hsa_circ_0001006 (circ_0001006) was upregulated in cardiac hypertrophy mice and cardiomyocytes treated with angiotensin II (Ang II). Next, we noticed that gain of function circ_0001006 could induce cardiomyocyte hypertrophy; oppositely, knockdown of circ_0001006 remitted Ang II-induced cardiomyocyte hypertrophy. Biotin-coupled miRNA and RNA-pull down assays showed that miR-214-3p could bind with circ_0001006 and gain the function of miR-214-3p abrogated the pro-hypertrophy effect of circ_0001006. Furthermore, Further, dual-luciferase reporter assay showed that miR-214-3p could interact with 3'UTRs of the PAK6 gene, and circRNA_0001006 could block the above interactions. Additionally, PAK6 expression is inhibited by miR-214-3p mimic in cardiomyocytes but enhanced by over-expression of circRNA_000203 in vitro.

Conclusions: Our data demonstrated that circRNA_0001006 exacerbates cardiac hypertrophy via suppressing miR-214-3p leading to enhanced PAK6 levels.

\section{INTRODUCTION}

Cardiac hypertrophy can be divided into physiological and pathological types related to normal cardiac function and impaired cardiac function $[1,2]$. Pathological hypertrophy of the myocardium is associated with several adverse cardiovascular events, including arrhythmias and heart failure. Studies have shown that chromatin remodeling and histone modification play essential roles in myocardial development and heart disease [3]. Non-coding RNAs are critical participants in cardiovascular physiological and pathological processes [4]. One of the non-coding RNAs, miRNAs, are 20-23 nucleotide RNAs that mediate various physiological functions and diseases by regulating target genes. Previous reports showed that 
miR-498 [5], miR-206 [6], miR-155 [7], and miR-451 [8] were all involved in cardiac hypertrophy progress.

Circular RNA (circRNA) serves as an essential class of non-coding RNA (ncRNA) and is mainly produced by reverse splicing of mRNA from the $3^{\prime}$ and $5^{\prime}$ end precursors of thousands of exons in eukaryotes $[9,10]$. Increasing evidence has identified that circRNAs possess the binding sites of miRNAs and function as sponges of miRNAs to regulate target genes $[11,12]$. CircRNA is involved in a variety of heart disease processes $[10,13]$. Pan RY et al. found that 66 circRNAs are involved in the regulation of coronary artery atherosclerosis and may be applied as the therapeutic or diagnostic markers $[14,15]$. ATP2A2 performed a ceRNA relationship with hsa_circ 0046159, which could regulate chronic thromboembolic pulmonary hypertension within the miR-1226-3p-circRNA regulatory network. [16]. CircHIPK3 promoted function of adrenaline via controlling miR-17-3p binding with ADCY6 during heart failure
[17]. In addition, CircFndc3b modulates FUS/VEGF-A axis after myocardial infarction and performs a protection effect [18].

Here, we performed that circ 0001006 was induced in cardiac hypertrophy mice and Ang II-treated cardiomyocytes, which displayed pro-hypertrophy effect by inhibiting miR-214-3p and demonstrated a mechanism of circ_0001006 via the miR-214-3p/PAK6 axis.

\section{RESULTS}

\section{Upregulation of circ 0001006 in cardiac hypertrophy mice and AngII-treated cardiomyocytes}

Firstly, the mice were randomly divided into two groups, including sham and TAC group. The TAC group performed the ventricles wall thickened. We also overserved the enhanced heart weight to body weight $(\mathrm{HW} / \mathrm{BW})$ ratio (Figure $1 \mathrm{~A}$ and $1 \mathrm{~B})$. We observed the

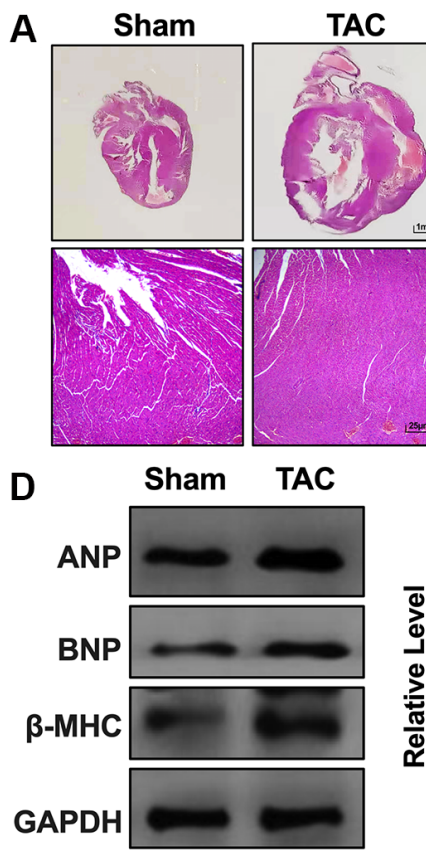

B

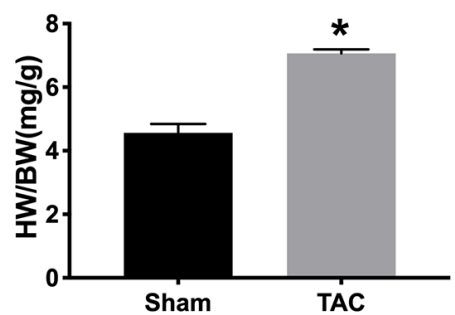

C

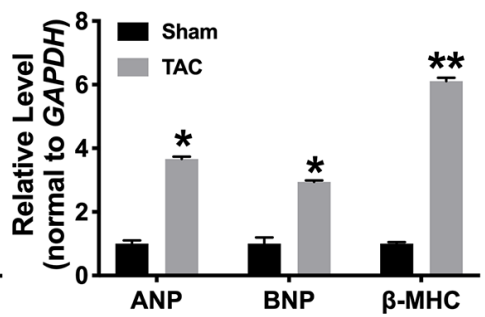

E

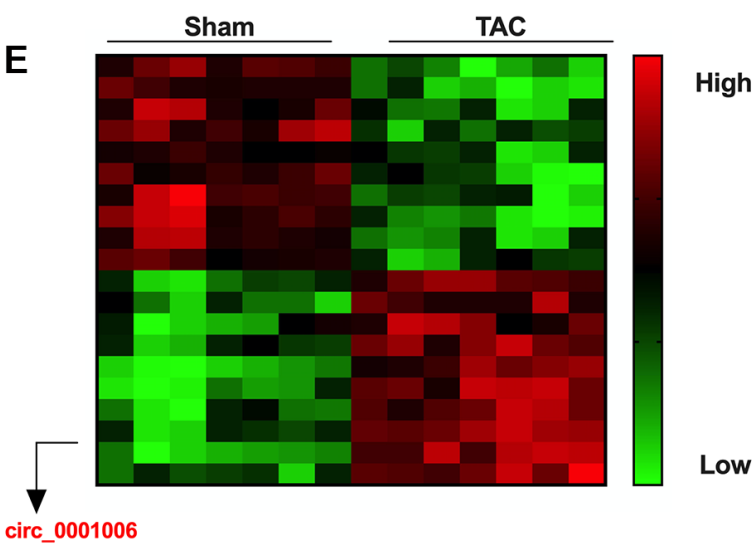

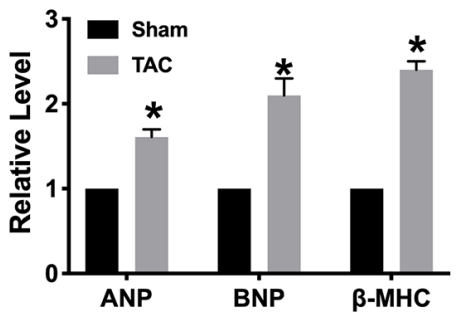

circ 0001006
F

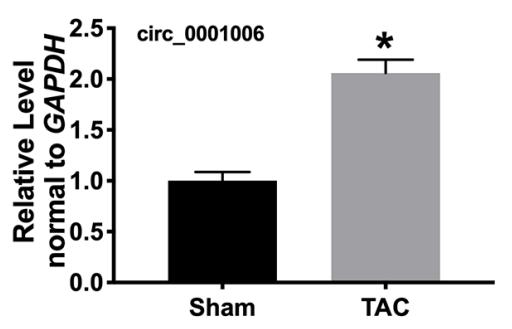

Figure 1. Upregulation of circ_0001006 in TAC induced cardiac hypertrophy. (A) Morphological experiment was performed to visualization the heart size (H\&E staining, Sham indicated sham-operated group, TAC indicated transverse aortic constriction operation group). (B) The histogram results of HW/BW ratios in a different group. (C) qRT-PCR assays of cardiac hypertrophy biomarker mRNA level, ANP, BNP, and B-MHC. (D) The expression of circ_0001006 in TAC and Sham mice. (E) The microarray of circRNAs in TAC and Sham mice tissues. (F) qRT-PCR assay analysis of the circ_0001006 expression level in heart tissues. ${ }^{*} P<0.05$ and ${ }^{* *} P<0.01$. All experiments were repeated at least three independent experiments. 
expression of the biomarkers indicating hypertrophy, such as ANP, BNP, and $\beta$-MHC, by RT-PCR and Western blot assays. The result showed an increased level in the TAC group compared with the sham group (Figure 1C and 1D). Then, a microarray was performed to screen the differential expression of circRNA. Among them, circ_0001006 was the most significant change in TAC tissues (Figure 1E). RT-PCR assays results were also performed that circ_0001006 was upregulated in the TAC group (Figure 1F).

Furthermore, A vitro model was used in this research. Cardiomyocytes were treated with Ang II $(200 \mathrm{nmol} / \mathrm{L})$ for $48 \mathrm{hrs}$, both cell cross size, hypertrophic biomarkers, and protein/DNA ratio increased remarkably in Ang IItreated cells (Figure 2A-2D). Consistent with results in vivo, circ_0001006 was upregulated in cardiomyocytes treated with Ang II (Figure 2E).

\section{Circ_0001006 induces hypertrophy of cardiomyocytes}

We transfected the constructed plasmid circ_0001006 and vector into cardiomyocytes, the efficiency of overexpression was about $51.22 \%$ (Figure 3A). Fluorescence assays displayed the enlarged cell size induced after transfection circ_0001006 (Figure 3B), overexpression of circ_0001006 resulted in increases in the protein/DNA ratio (Figure 3C). Furthermore, the hypertrophic markers were all increasing compared with the vector group, which indicated the pro-hypertrophy effect of cardiomyocytes (Figure 3D and 3E). In summary, overexpression of circ_0001006 would induce cardiac hypertrophy.

\section{Knockdown of circ_0001006 remits myocardial hypertrophy}

Then, we would like to determine whether the loss function of circ_0001006 performs inhibition function on cardiac hypertrophy, and we, therefore, created short hairpin RNA (shRNA) sh-circ_0001006 or sh-Scramble (sh-Scr) and transfected into cardiomyocytes treated with Ang II (200 nmol/L) for $48 \mathrm{hrs}$ (Figure 4A). The cell surface area was performed by immunofluorescence (Figure 4B), loss function of circ_0001006 decreased cell cross area and the protein/DNA ratio after disposing of Ang II (Figure 4C), the levels of hypertrophic markers were also reduced compared with the sh-Scr group (Figure 4D and 4E).

\section{MiR-214-3p/PAK6 may be downstream of circ_0001006}

Bioinformatics website assays revealed that miR-214$3 \mathrm{p}$ interacted with circ_0001006 (Figure 5A). To identify the association of miR-214-3p with circ_0001006, we conducted RNA pull down assays using a circ_0001006-specific probe. circ_0001006 precipitation complex performed the enrichment of
A

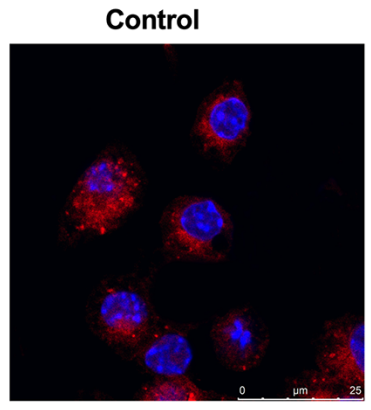

C

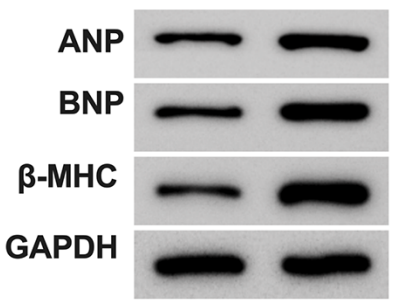

Angll
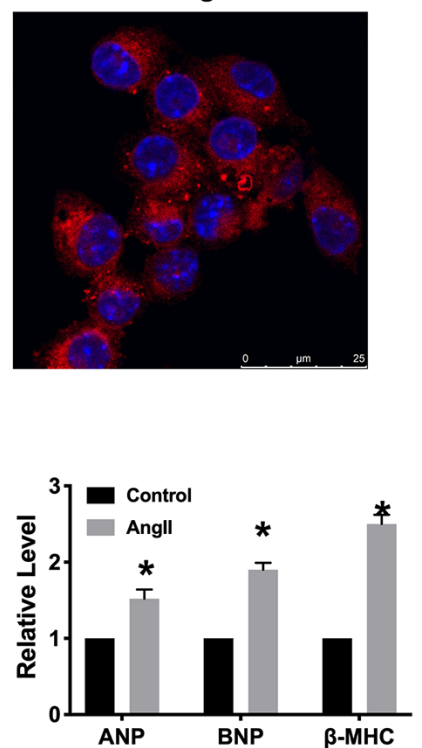

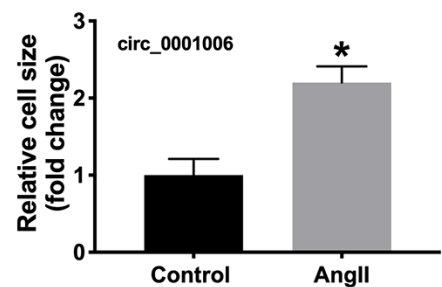

B

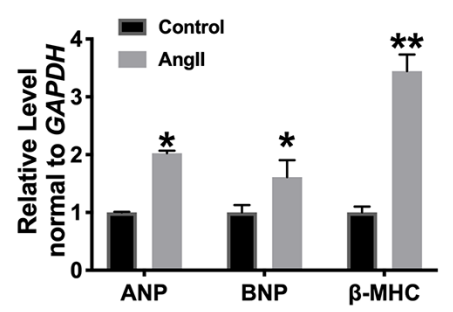

$E$
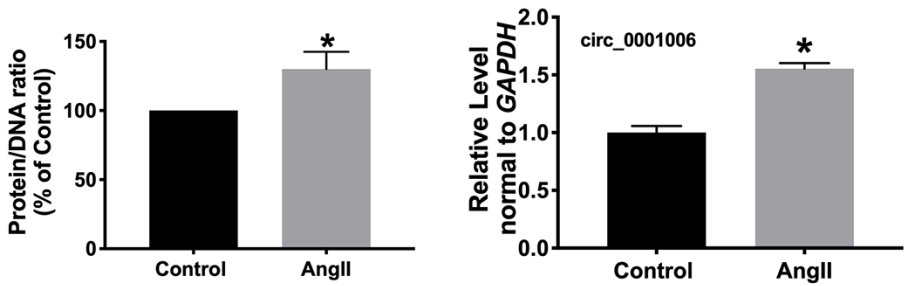

Figure 2. circ_0001006 was upregulated in Angll-induced cardiomyocyte hypertrophy. (A) The immunofluorescence results of cardiomyocytes treated with or without Ang II. ( $\alpha$-actinin, red, DAPI, blue, The scale bar is $25 \mu \mathrm{m}$ ). (B) qRT-PCR assay analysis of the circ_0001006 expression level in cardiomyocytes. (C) Western blot analysis of ANP, BNP, $\beta$-MHC expression in cardiomyocytes. (D) Protein/DNA ratio of the cardiomyocytes. (E) The expression of circ_0001006 in vitro. ${ }^{*} P<0.05$ and ${ }^{* *} P<0.01$. Data are shown as mean \pm SEM. All experiments were repeated at least three independent experiments. 
A

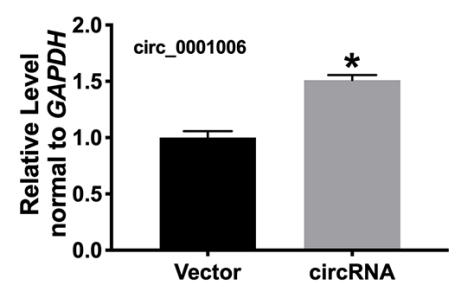

C

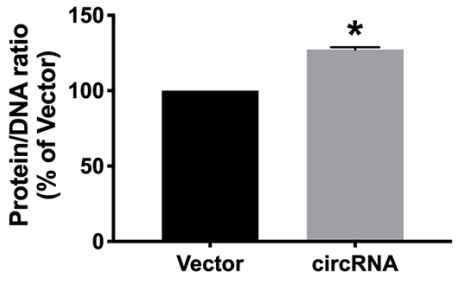

B

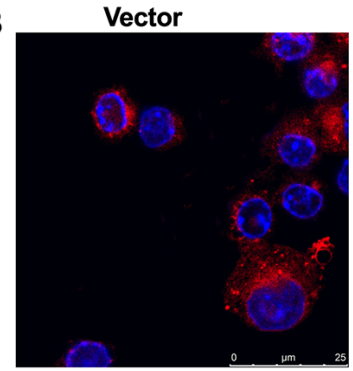

D

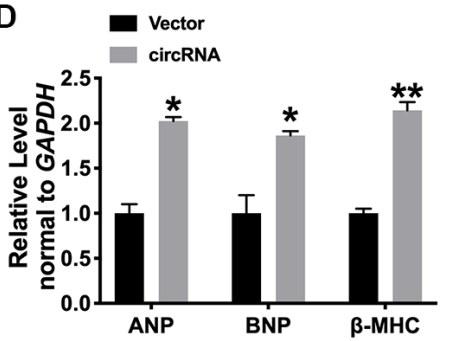

circRNA

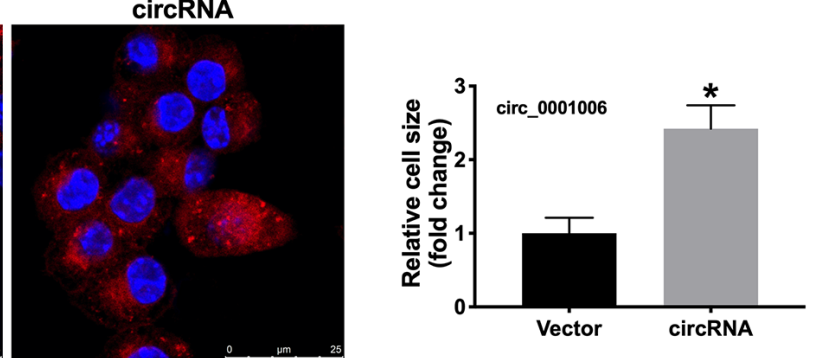

E

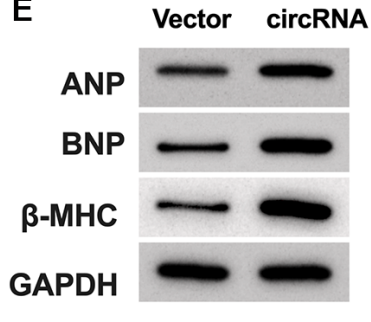

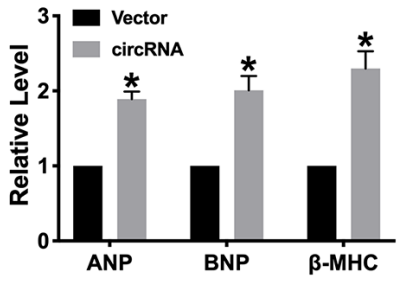

Figure 3. circ_0001006 induced hypertrophic in cardiomyocytes. (A) The level of circ_0001006 in cardiomyocytes after transfecting circ_0001006/Vector. (B) Representative immunofluorescence results of cardiomyocytes after transfecting circ_0001006/Vector (The scale bar is $25 \mu \mathrm{m}$ ). (C) Protein/DNA ratio of the cardiomyocytes after transfecting circ_0001006/Vector. (D) The mRNA level of ANP, BNP, and $\beta$ MHC in cardiomyocytes. (E) Western blot assay of ANP, BNP, $\beta$-MHC protein level in cardiomyocytes. ${ }^{*} P<0.05$ and ${ }^{* *} P<0.01$. Data are shown as mean \pm SEM. All experiments were repeated at least three independent experiments.

A

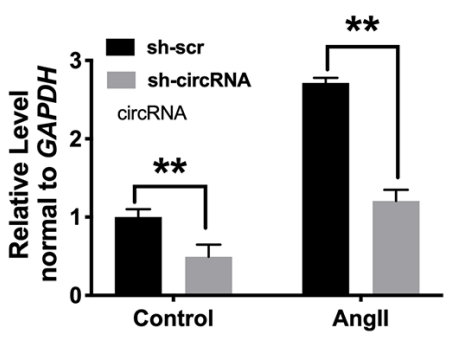

C

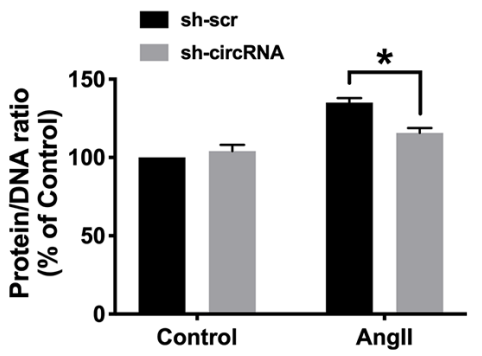

B

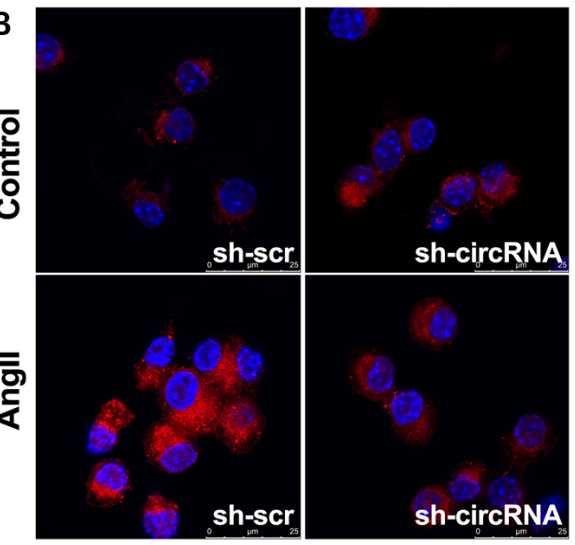

$E$

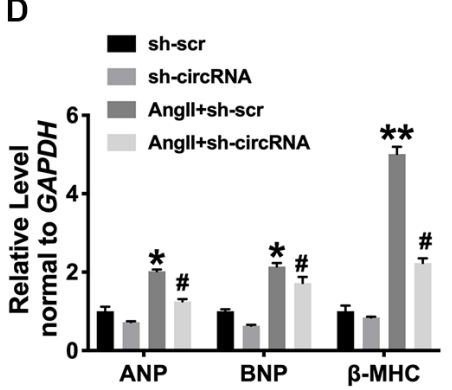

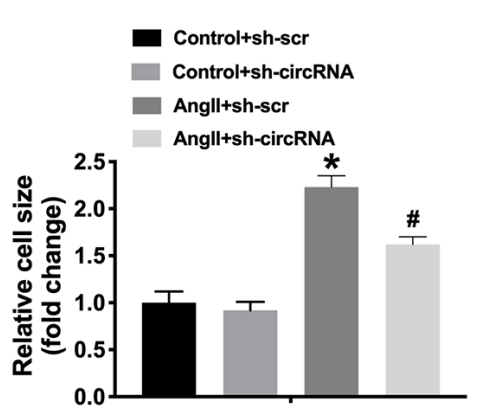

$\beta-M H C$ GAPDH

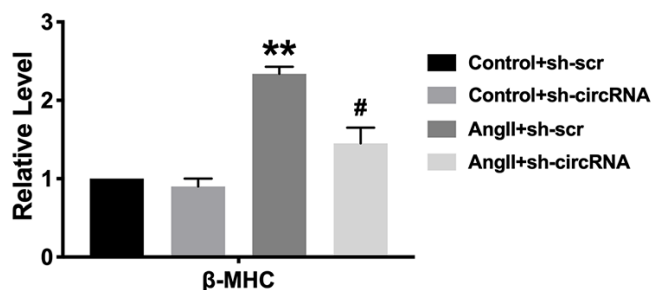

Figure 4. Silencing of circ_0001006 prevents cardiomyocyte hypertrophy. (A) The transfection efficiency of sh-circ_0001006 was verified. (B) Representative immunofluorescence results performed the effect of knocking down circ_0001006 (The scale bar is $25 \mu \mathrm{m}$ ). (C) Protein/DNA ratio of the cardiomyocytes after transfecting with sh-circ_0001006/sh-Scr treated with or without Ang II. (D) ANP, BNP, and $\beta$ $\mathrm{MHC}$ expression level in cardiomyocytes. (E) The expression of $\beta$-MHC in cardiomyocytes. ${ }^{*} P<0.05$ and ${ }^{* *} P<0.01$ vs. Control+sh-scr group, ${ }^{\#} P$ $<0.05$ vs. Control+sh-circRNA. Data are shown as mean \pm SEM. All experiments were repeated at least three independent experiments. 
miR-214-3p (Figure 5B and 5C). Luciferase assay also confirmed that miR-214-3p could interact with circ_0001006 (Figure 5D). Then we found that the level of miRNA-214-3p was upregulated after transfecting shcirc 0001006 and downregulation after overexpression circ_0001006 (Figure 5E and 5F). These results performed that circ_0001006 could bind to miR-214-3p.

PAK family is divided into two categories. I class PAK1-3, II class contains PAK4-6. Previous reports showed that mice knockout of PAK1 promote isoproterenol-induced cardiac hypertrophy, which could activate Erk1/2 and inhibit protein phosphatase 2A [19]. PAK family members participated in severe heart disease progression [20], and we observed that PAK6 possessed the potential binding site of miR-214-3p by using Target Scan Human 7.2 bioinformatics website
(Figure 5G). The luciferase report verified that miR214-3p could interact with and inhibit the expression of PAK6 (Figure 5G). Further, miR-214-3p reduced PAK6 expression, and circ_0001006 could induce the expression of PAK6 (Figure 5H-5K).

\section{Circ_0001006 regulates hypertrophy by targeting miR-214-3p/PAK6}

Next, we found the decreased level of miR-214-3p and increased level of PAK6 in AngII-treated cardiomyocytes (Figure 6A). Furthermore, we cotransfection circ_0001006 with miRNA-214-3p mimics or si-PAK6 in normal cardiomyocytes. The gain function of circ 0001006 increases the cell cross size and the protein/DNA ratio in Ang II-treated cardiomyocytes, which were remitted by miRNA-214-
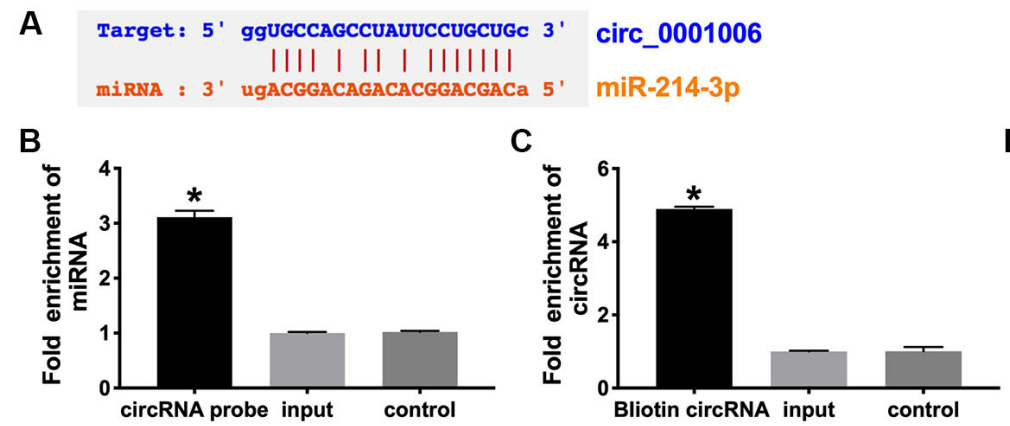

D

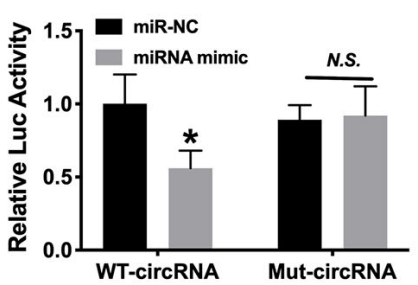

E

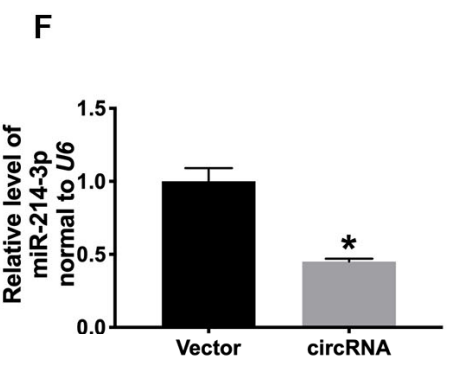

\section{G}

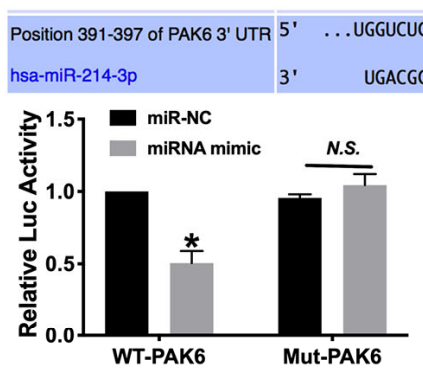

H

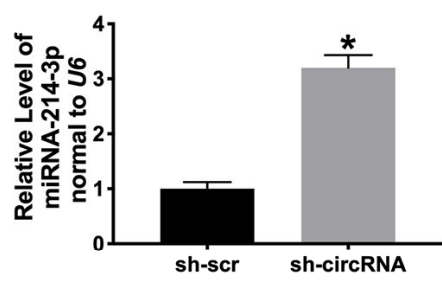

I

J

K
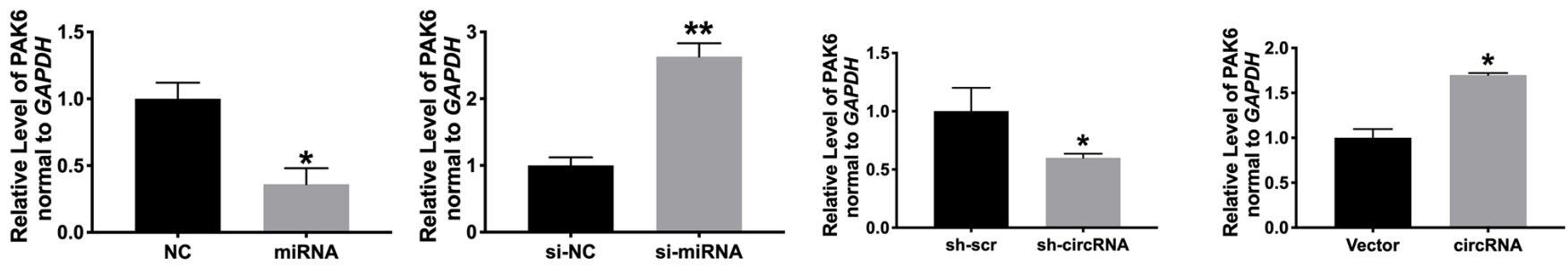

Figure 5. MiR-214-3p/PAK6 may be downstream of circ_0001006. (A) Bioinformatics website forecast revealed that circ_0001006 includes site binding to miR-214-3p provided by predication tools (http://starbase.sysu.edu.cn/index.php). (B) circ_0001006 in cell lysis was pulled down and collected with a circ_0001006-specific probe and then measured using qRT-PCR. miR-214-3p was pulled down and collected with a circ_0001006-specific probe and then evaluated using qRT-PCR. (C) Luciferase assay report verified the relationship between circ_0001006 and miR-214-3p. ( $D$ and $\mathbf{E}$ ). the expression of miR-214-3p in cardiomyocytes after sh-circRNA and circRNA transfection. (F) Luciferase assay report verified the relationship between PAK6 and miR-214-3p. (G-K). The expression of PAK6 was detected in cardiomyocytes. Data are shown as mean \pm SEM. ${ }^{*} P<0.05$ and ${ }^{* *} P<0.01$. All experiments were repeated at least three independent experiments. 
3p mimics or si-PAK6 (Figure 6B and 6C). The mRNA levels of hypertrophy biomarkers were also recovered by overexpression miRNA-214-3p or si-PAK6 (Figure 6D). Co-transfection of PAK6 abolished the function of miR214-3p on Ang II-treated cardiomyocytes, which was performed by promoting hypertrophic markers expression (Figure 6E-6G). Meanwhile, silencing of circ_0001006 inhibited cardiomyocyte hypertrophy, which was abolished by si-miR-214-3p or PAK6 overexpression (Figure 6H-6J). Taken together, circ_0001006 regulates hypertrophy by targeting miR-214-3p/PAK6.

\section{Circ_0001006 prevents cardiac hypertrophy in vivo}

Then, AAV9 sh-circ_0001006 or AAV9 sh-Scr were injected into mice via tail vein for 21 days, and the mice have subjected to TAC surgery for creating cardiac hypertrophy model. The cardiac function was determined by echocardiography. AAV9 sh-circ_0001006 recovered the EF (\%) and FS (\%) in TAC mice (Figure 7A and 7B). The results showed a lower HW/BW ratio in the shcirc 0001006 injection TAC mice (Figure 7C). The $\mathrm{ANP}, \mathrm{BNP}$, and $\beta$-MHC levels were dramatically downregulated when compared with the sh-Scr group in TAC mice (Figure 7D). H\&E staining results performed that AAV9 sh-circ_0001006 prevented cardiac hypertrophy in TAC mice (Figure 7E). We performed qRT-PCR assays to verify the downregulation of circ_0001006 (Figure 7F). We also performed the change of miR-241-3p and PAK6 level was consistent with the results in vitro (Figure $7 \mathrm{G}$ and $7 \mathrm{H}$ ). Together, these data suggest that circ_0001006 promoted cardiac hypertrophy via suppressing miR-214-3p targeting to PAK6.

\section{DISCUSSION}

In conclusion, our research demonstrated that circ_0001006 could promote cardiac hypertrophy by
A

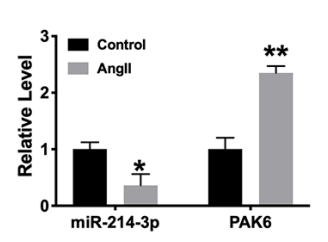

D

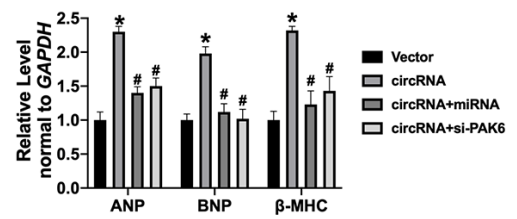

G

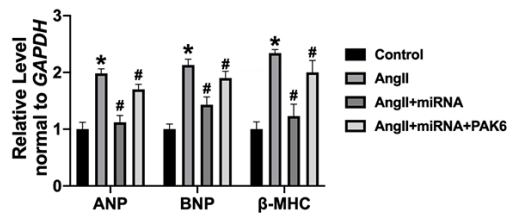

J

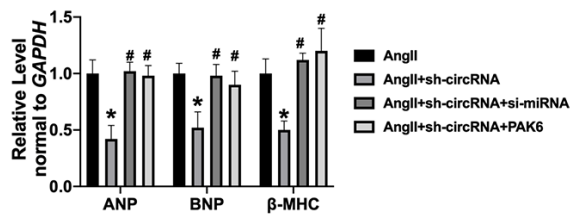

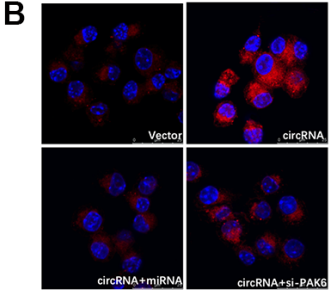

$\mathrm{E}$

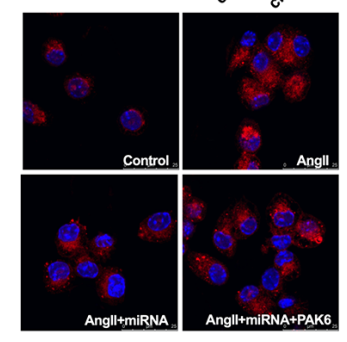

H
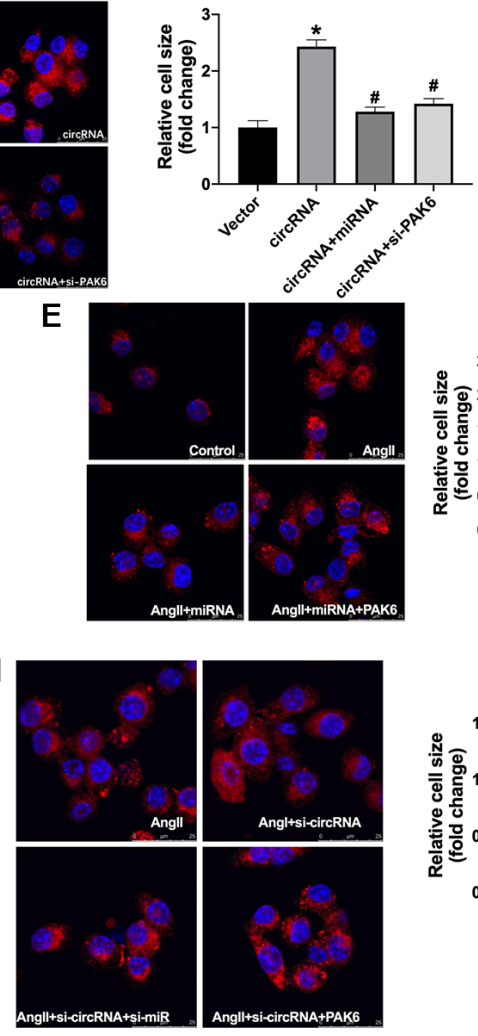

C

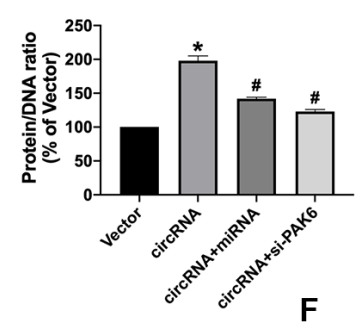

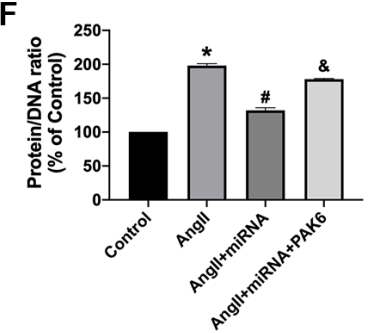

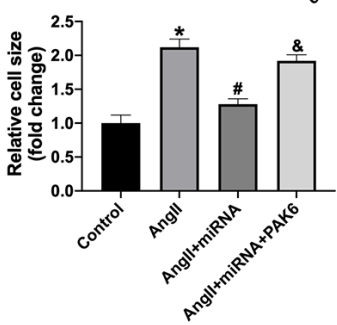

I
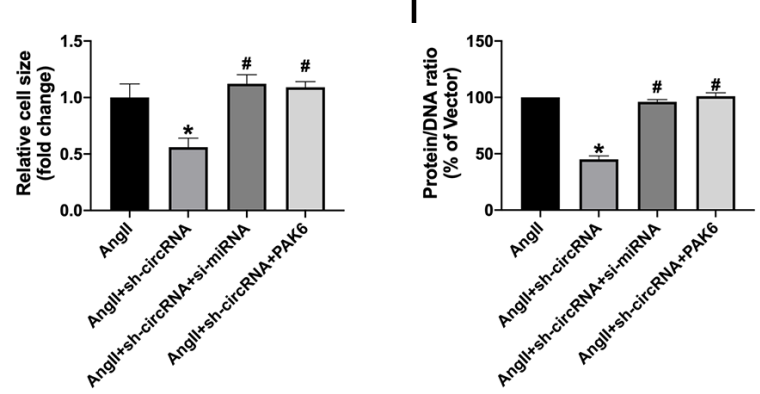

Figure 6. The circ_0001006 regulates cardiac hypertrophy via suppressing miR-214-3p/PAK6. (A) qRT-PCR assay analysis of PAK6 and miR-214-3p level in cardiomyocytes. (B) Representative immunofluorescence results of cardiomyocytes (The scale bar is $25 \mu \mathrm{m}$ ). (C) Protein/DNA ratio of the cardiomyocytes. (D) The expression of ANP, BNP, and $\beta$-MHC in cardiomyocytes. (E) Representative immunofluorescence results of cardiomyocytes (The scale bar is $25 \mu \mathrm{m}$ ). (F) Protein/DNA ratio of the cardiomyocytes. (G) The expression of ANP, BNP, and $\beta-M H C$ in cardiomyocytes. (H) Representative immunofluorescence results of cardiomyocytes (The scale bar is $25 \mu \mathrm{m}$ ). (I) Protein/DNA ratio of the cardiomyocytes. (J) The expression of ANP, BNP, and $\beta$-MHC in cardiomyocytes. Data are shown as mean \pm SEM. ${ }^{*} P$ and $^{\#} P<0.05$ and $^{* *} P<0.01$. All experiments were repeated at least three independent experiments. 
decreasing miR-214-3p via targeting PAK6, which is a novel mechanism in cardiac hypertrophy. Our findings may provide a prediction and diagnosis method for pathological cardiac hypertrophy.

Pressure load-induced cardiac hypertrophy was mediated by different signaling pathways. Signaling pathways are numerous, link between each other, and form a complex network. Exploring the occurrence and development of cardiomyocyte hypertrophy is of profound significance for the prevention and treatment of myocardial hypertrophy.

In this research, we found that circ 0001006 was markedly upregulated in cardiac hypertrophy. We firstly investigated the role of cardiomyocytes with the gain or loss function of circ 0001006. We found that the gain function of circ_0001006 induced hypertrophy in cardiomyocytes, knockdown of circRNA performed opposite results. We demonstrated that circ_0001006 interacted with miR-214-3p to induce the expression of in cardiomyocytes. The present study revealed that circ_0001006 could induce cardiac hypertrophy via inhibiting miR-214-3p binding to PAK6.

Due to its unique molecular biological characteristics, circRNA plays a crucial role in the diagnosis of a variety of diseases. However, the fact that circRNAs are highly maladjusted in condition and exhibit a high degree of tissue and disease specificity makes them ideal candidates for disease diagnosis. CircRNA also plays a decisive function in heart disease. It was reported that circRNA_000203 suppressed miR-26b$5 p / m i R-140-3 p$ to abolish the inhibition of Gata4, which aggravates cardiac hypertrophy [21]. As an endogenous sponge, circSlc8a1 can interact with miR-133a in cardiomyocytes, which indicated circSlc8al might play a key role in cardiac hypertrophy [22].

In previous reports, circ_0001006 was identified as a potential biomarker and therapeutic target in breast cancer brain metastasis [23]. In the plasma of gastric cancer patients, circ_0001006 may be used as diagnostic indicators due to anomalous change [24]. Up to now, studies of circRNA in heart disease, especially in cardiac hypertrophy, are limited, which requires us to explore the characteristics and mechanisms further. Our data report that circ_0001006 interacted with miR-214$3 p$. Functionally, circ_0001006 could promote the increases of cell cross area and the level of biomarkers, ANP, BNP, and $\beta$-MHC in Ang II-treated cardiomyocytes by targeting miR-214-3p.

Our current results have provided proof to sustain the viewpoint that miR-214-3p suppresses cardiac hypertrophy by repressing PAK6. TargetScan website analyses performed that PAK6 may possess the binding site of miR-214-3p, for further verify the supposes,
A

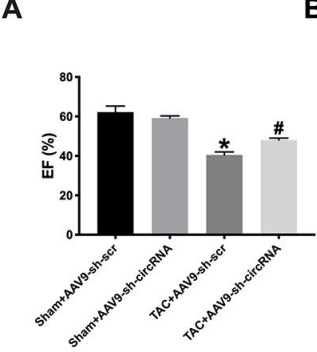

E

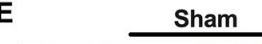

B

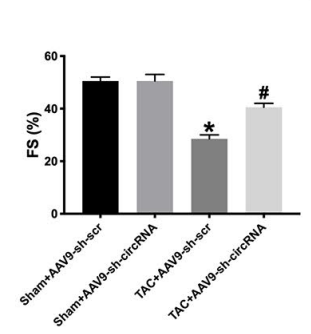

C

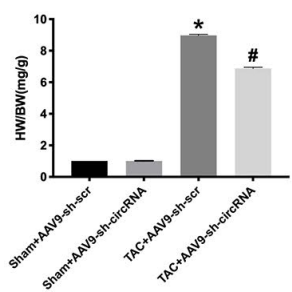

$\mathbf{F}$
D

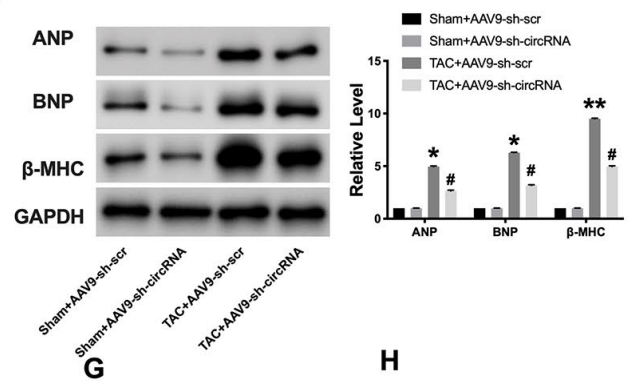

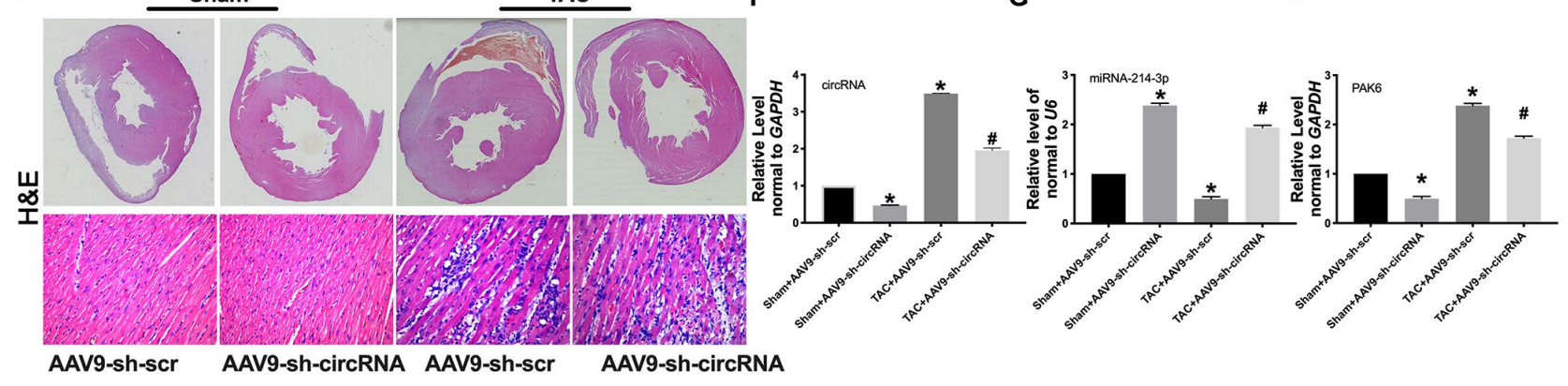

Figure 7. Knockdown of circRNA_0001006 alleviated cardiac hypertrophy in vivo. (A and B) EF (\%) and FS (\%) were assessed by echocardiography. (C) The histogram results of HW/BW ratios in different groups. (D) Western blot assay analysis of ANP, BNP, and $\beta$-MHC expression level in the tissue. (E) Representative histological results of the ( $\mathbf{H}$ and $\mathbf{E}$ ) Staining of the mouse heart tissues. (F) The AAV9 transfection efficiency of circ_0001006 in the mouse heart. (G) The miR-214-3p level in heart tissue. (H) The mRNA level of PAK6 in mice. ${ }^{*} P<$ 0.05 and ${ }^{* *} P<0.01$ vs. Sham+AAV9-sh-scr group, ${ }^{\#} P<0.05$ vs. Sham+AAV9-sh-circRNA group. Data are shown as mean \pm SEM. All experiments were repeated at three independent experiments. 
luciferase report was conducted to perform miR-214-3p binding with 3'-UTRs of PAK6. The gain function of miR-214-3p repressed transcriptional level of PAK in cardiomyocytes. Then, PAK6 inhibited the antihypertrophy effect of miR-214-3p in cardiomyocytes, the conclusion showing that PAK6 participated in miR214-3p-mediated cardiac hypertrophy progress.

\section{METHODS}

\section{Animal models}

All mice were fed under standard conditions, with standard food and water. Adult male C57BL/6 mice ( $\sim 25 \mathrm{~g}, 8$ weeks) previously described were used in this study [25]. Cardiac hypertrophy was induced by TAC (transverse aortic constriction) operation. The similar surgery without ligation was conducted in the sham group. Then the mice were fed for 4 weeks. Before 1 week of TAC surgery, the animals were injected with AAV9 sh-Scr or sh-circ_0001006 (Biocobio, Tianjin, China) was injected in the left ventricle myocardium. The effect of AAV9 reaches highest about 3 weeks. The animal study was reviewed and approved by Baotou Medical College.

\section{Microarray assay}

TRIzol reagent (Invitrogen) was used to extract total RNAs from heart tissues, followed by the quantified analysis (NanoDrop). After the standard protocol of Arraystar, we conducted the microarray hybridization. Briefly, fluorescent complementary RNAs (cRNAs) were produced from the purified RNAs and hybridized in circRNA array. The arrays were scanned by Agilent Scanner G2505C and the images and the data were analyzed by gilent Feature Extraction software (version 11.0.1.1) and $\mathrm{R}$ software, respectively. The differently expressed circRNAs were shown in the heat map based on the criteria (fold change $>2$ and $P<0.05$ ).

\section{Echocardiography}

The cardiac function was analyzed by echocardiography measurement with a Vevo 2100 Imaging System furnished with a $30 \mathrm{MHz}$ phased-array in mice with TAC for 8 weeks. Diastolic and systolic volumes were acquired by applying Simpson's rule of discs to the serially acquired short-axis images (Table 1).

\section{Cell culture and treatment}

Cardiomyocytes were collected from neonatal mice (13 days) [26]. Digesting the cardiac tissue with trypsin (Solarbio, Beijing, China), and cardiomyocytes were cultured at $37^{\circ} \mathrm{C}$ with $5 \% \mathrm{CO}_{2}$ in DMEM (Waltham,
USA) containing FBS (10\%, Cromwell, USA) and penicillin/streptomycin $(100 \mu \mathrm{L} / \mathrm{mL}$, Sigma-Aldrich, USA). For inducing cell hypertrophy, the cardiomyocytes were disposed of with angiotensin II (Ang II, $200 \mathrm{nmol} / \mathrm{L}$ ) for $48 \mathrm{hrs}$.

\section{Immunostaining}

Cells were fixed with $14 \%$ formaldehyde and embedded in paraffin. Immunohistochemical staining was performed according to the instructions of the primary antibody ( $\alpha$-actinin, Abcam, Cambridge, UK). The nucleus was stained using DAPI. The images were observed by an optical microscope (Olympus, Japan).

\section{Protein/DNA ratio detection}

The cells were washed using PBS and subjected in the cellular protein and DNA content quantitative analysis. The sample was centrifuged $(10,000 \mathrm{~g}, 10$ minutes $)$ after the treatment of $0.2 \mathrm{~N}$ perchloric acid $(1 \mathrm{ml})$, followed by the incubation of $\mathrm{NKOH}\left(250 \mathrm{ml}, 0.3 \mathrm{~N}, 60^{\circ} \mathrm{C}, 20\right.$ minutes). The Lowry method was used to analyze protein content, in which serum albumin served as the standard. The Hoechst dye 33258 was used to analyze DNA content, in which salmon sperm DNA served as the standard.

\section{Quantitative real-time PCR}

Cells were transfected with vectors and/or oligonucleotides indicated in each experiment. RNA, and were treated with Trizol reagent (Sigma, USA) to isolate total RNA. The RNA was reverse-transcript to cDNA by using a superscript reverse transcriptase (Invitrogen, Carlsbad, CA), and quantified by a ViiA7 Quantitative PCR System (Applied Biosystems, Carlsbad, CA). The relative change of RNA levels was calculated by $2^{-\Delta \Delta C T}$ method and normalized to GAPDH or U6.

\section{Dual-Luciferase reporter assay}

The wild type sequences or mutant sequence of circ_0001006 and 3'UTR region of PAK6 were cloned into the pmirGLO vectors (Promega, USA). The cells were transfected with circ 0001006-WT and Mut, or PAK6-WT and Mut, along with miR-214-3p or NC. After 48 hours incubation, the cells were lysed and the luciferase intensity was detected by a Dual Luciferase assay kit (Biovision, China).

\section{Western blot analysis}

Cells with the indicated treatment were lysed by icecold RIPA buffer (Thermo, USA) added with a cocktail of protease inhibitors (Thermo). The lysates were then 
Table 1. Comparison of left ventricular echocardiographic parameters between the two groups.

\begin{tabular}{lcc}
\hline \multirow{2}{*}{ Index } & \multicolumn{2}{c}{ 8 weeks } \\
\cline { 2 - 3 } & Sham & TAC \\
\hline LVIDs & $3.08 \pm 0.21$ & $4.09 \pm 0.13$ \\
LVIDd & $3.79 \pm 0.23$ & $4.92 \pm 0.21$ \\
LVPWd & $0.58 \pm 0.03$ & $0.92 \pm 0.03$ \\
EF & $54.23 \pm 3.73$ & $38.79 \pm 3.73$ \\
FS & $32.23 \pm 4.23$ & $23.79 \pm 2.23$ \\
\hline
\end{tabular}

subjected to the SDS-PAGE and shifted to nitrocellulose membranes (absin, China). The protein bands were interacted with primary antibodies at room temperature for 2 hours and $4^{\circ} \mathrm{C}$ overnight, followed by secondary anti-mouse and anti-rabbit antibodies. The bands were then visualized by using an Odyssey in an Odyssey software (LI-COR, USA).

\section{Histological analysis}

The heart tissue was gathered and fixed in $4 \%$ paraformaldehyde for $24 \mathrm{hrs}$. Then the fixed tissues were embedded in paraffin. Next, a Paraffin slicer machine was used to cut slices (5- $\mu \mathrm{m}$ cross-sectional). $\mathrm{H} \& \mathrm{E}$ staining was used to evaluate cardiac morphology. The results were calculated with image $\mathrm{J}$ 6.0.

\section{Biotin-coupled circRNA and miRNA enrichment}

Biotin-coupled miRNA and circRNA pull-down assays were executed according to the protocol [27, 28]. Briefly, HEK293 cells were transfected with $3^{\prime}$ end biotinylated circ_0001006 (20 nM) for 1 day. 4 MyOne streptavidin C1 Dynabeads was carried out to pull down biotin-coupled RNA complexes. The enrichment of circ_0001006 or miR-214-3p was measured by PCR.

\section{Statistical analysis}

All data is statistical as a mean \pm S.E.M. We performed Student's $t$-test or a one-way ANOVA for statistical analysis. $P<0.05$ was described as statistically significant.

\section{AUTHOR CONTRIBUTIONS}

Xuefeng Lin and Liqin Zhang designed the experiments. Wei Zhang and Xinjun Lei performed the experiments and analyzed the data. Qun Lu and Aiqun Ma wrote the paper.

\section{CONFLICTS OF INTEREST}

The authors declare no conflicts of interest related to this study.

\section{REFERENCES}

1. Frieler RA, Mortensen RM. Immune cell and other noncardiomyocyte regulation of cardiac hypertrophy and remodeling. Circulation. 2015; 131:1019-30. https://doi.org/10.1161/CIRCULATIONAHA.114.008788 PMID:25779542

2. Heineke J, Molkentin JD. Regulation of cardiac hypertrophy by intracellular signalling pathways. Nat Rev Mol Cell Biol. 2006; 7:589-600. https://doi.org/10.1038/nrm1983 PMID:16936699

3. Li Y, Liang $Y$, Zhu Y, Zhang Y, Bei Y. Noncoding RNAs in Cardiac Hypertrophy. J Cardiovasc Transl Res. 2018; 11:439-49.

https://doi.org/10.1007/s12265-018-9797-x PMID: $\underline{0171598}$

4. Heggermont WA, Papageorgiou AP, Quaegebeur A, Deckx S, Carai P, Verhesen W, Eelen G, Schoors S, van Leeuwen R, Alekseev S, Elzenaar I, Vinckier S, Pokreisz $P$, et al. Inhibition of MicroRNA-146a and Overexpression of Its Target Dihydrolipoyl Succinyltransferase Protect Against Pressure Overload-Induced Cardiac Hypertrophy and Dysfunction. Circulation. 2017; 136:747-61. https://doi.org/10.1161/CIRCULATIONAHA.116.024171 PMID:28611091

5. Wang K, Liu F, Zhou LY, Long B, Yuan SM, Wang Y, Liu $C Y$, Sun $T$, Zhang $X J$, Li PF. The long noncoding RNA CHRF regulates cardiac hypertrophy by targeting miR489. Circ Res. 2014; 114:1377-88.

https://doi.org/10.1161/CIRCRESAHA.114.302476 PMID:24557880

6. Yang $Y$, Del Re DP, Nakano N, Sciarretta S, Zhai P, Park J, Sayed D, Shirakabe A, Matsushima S, Park Y, Tian B, Abdellatif M, Sadoshima J. miR-206 Mediates YAPInduced Cardiac Hypertrophy and Survival. Circ Res. 2015; 117:891-904. https://doi.org/10.1161/CIRCRESAHA.115.306624 PMID:26333362

7. Seok HY, Chen J, Kataoka M, Huang ZP, Ding J, Yan J, Hu X, Wang DZ. Loss of MicroRNA-155 protects the 
heart from pathological cardiac hypertrophy. Circ Res. 2014; 114:1585-95. https://doi.org/10.1161/CIRCRESAHA.114.303784 PMID:24657879

8. Kuwabara Y, Horie T, Baba O, Watanabe S, Nishiga M, Usami S, Izuhara M, Nakao T, Nishino T, Otsu K, Kita T, Kimura T, Ono K. MicroRNA-451 exacerbates lipotoxicity in cardiac myocytes and high-fat dietinduced cardiac hypertrophy in mice through suppression of the LKB1/AMPK pathway. Circ Res. 2015; 116:279-88.

https://doi.org/10.1161/CIRCRESAHA.116.304707 PMID:25362209

9. Kristensen LS, Andersen MS, Stagsted LVW, Ebbesen KK, Hansen TB, Kjems J. The biogenesis, biology and characterization of circular RNAs. Nat Rev Genet. 2019; 20:675-91.

https://doi.org/10.1038/s41576-019-0158-7 PMID:31395983

10. Beermann J, Piccoli MT, Viereck J, Thum T. Noncoding RNAs in Development and Disease: Background, Mechanisms, and Therapeutic Approaches. Physiol Rev. 2016; 96:1297-325. https://doi.org/10.1152/physrev.00041.2015 PMID:27535639

11. Verduci L, Strano S, Yarden Y, Blandino G. The circRNA-microRNA code: emerging implications for cancer diagnosis and treatment. Mol Oncol. 2019; 13:669-80.

https://doi.org/10.1002/1878-0261.12468 PMID: $\underline{30719845}$

12. Dori $M$, Bicciato $S$. Integration of Bioinformatic Predictions and Experimental Data to Identify circRNAmiRNA Associations. Genes (Basel). 2019; 10:642. https://doi.org/10.3390/genes10090642 PMID:31450634

13. Lim TB, Lavenniah A, Foo RS. Circles in the heart and cardiovascular system. Cardiovasc Res. 2020; 116:269-78.

https://doi.org/10.1093/cvr/cvz227 PMID: $\underline{31552406}$

14. Pan RY, Zhao $\mathrm{CH}$, Yuan JX, Zhang YJ, Jin JL, Gu MF, Mao ZY, Sun HJ, Jia QW, Ji MY, Zhang J, Wang LS, Ma WZ, et al. Circular RNA profile in coronary artery disease. Am J Transl Res. 2019; 11:7115-25.

PMID:31814914

15. Huang S, Li X, Zheng H, Si X, Li B, Wei G, Li C, Chen Y, Chen $Y$, Liao W, Liao $Y$, Bin J. Loss of Super-EnhancerRegulated circRNA Nfix Induces Cardiac Regeneration After Myocardial Infarction in Adult Mice. Circulation. 2019; 139:2857-76. https://doi.org/10.1161/CIRCULATIONAHA.118.038361 PMID: $\underline{0947518}$
16. Miao R, Gong J, Zhang C, Wang Y, Guo X, Li J, Yang S, Kuang T, Zhong J, Feng H. Hsa_circ_0046159 is involved in the development of chronic thromboembolic pulmonary hypertension. J Thromb Thrombolysis. 2020; 49:386-94.

https://doi.org/10.1007/s11239-019-01998-4

PMID: 31776848

17. Deng $Y$, Wang J, Xie G, Zeng X, Li H. Circ-HIPK3 Strengthens the Effects of Adrenaline in Heart Failure by MiR-17-3p - ADCY6 Axis. Int J Biol Sci. 2019; 15:2484-96.

https://doi.org/10.7150/ijbs.36149

PMID: 31595165

18. Garikipati VNS, Verma SK, Cheng Z, Liang D, Truongcao MM, Cimini M, Yue Y, Huang G, Wang C, Benedict C, Tang Y, Mallaredy V, Ibetti J, et al. Circular RNA CircFndc3b modulates cardiac repair after myocardial infarction via FUS/VEGF-A axis. Nat Commun. 2019; 10:4317.

https://doi.org/10.1038/s41467-019-11777-7 PMID:31541092

19. Taglieri DM, Monasky MM, Knezevic I, Sheehan KA, Lei M, Wang X, Chernoff J, Wolska BM, Ke Y, Solaro RJ. Ablation of p21-activated kinase- 1 in mice promotes isoproterenol-induced cardiac hypertrophy in association with activation of Erk1/2 and inhibition of protein phosphatase 2A. J Mol Cell Cardiol. 2011; 51:988-96.

https://doi.org/10.1016/j.yjmcc.2011.09.016 PMID:21971074

20. Quan L, Cheng Z, Dai Y, Jiao Y, Shi J, Fu L. Prognostic significance of PAK family kinases in acute myeloid leukemia. Cancer Gene Ther. 2020; 27:30-37. https://doi.org/10.1038/s41417-019-0090-1 PMID: $\underline{30890765}$

21. Li H, Xu JD, Fang XH, Zhu JN, Yang J, Pan R, Yuan SJ, Zeng $\mathrm{N}$, Yang ZZ, Yang $\mathrm{H}$, Wang XP, Duan JZ, Wang S, et al. Circular RNA circRNA_000203 aggravates cardiac hypertrophy via suppressing miR26b-5p and miR-140-3p binding to Gata4. Cardiovasc Res. 2020; 116:1323-34.

https://doi.org/10.1093/cvr/cvz215

PMID:31397837

22. Lim TB, Aliwarga E, Luu TDA, Li YP, Ng SL, Annadoray L, Sian S, Ackers-Johnson MA, Foo RS. Targeting the highly abundant circular RNA circSlc8a1 in cardiomyocytes attenuates pressure overload induced hypertrophy. Cardiovasc Res. 2019; 115:1998-2007. https://doi.org/10.1093/cvr/cvz130 PMID: $\underline{31114845}$

23. Fu B, Zhang A, Li M, Pan L, Tang W, An M, Liu W, Zhang J. Circular RNA profile of breast cancer brain metastasis: identification of potential biomarkers 
and therapeutic targets. Epigenomics. 2018; 10:1619-30.

https://doi.org/10.2217/epi-2018-0090

PMID:30810051

24. Li T, Shao Y, Fu L, Xie Y, Zhu L, Sun W, Yu R, Xiao B, Guo J. Plasma circular RNA profiling of patients with gastric cancer and their droplet digital RT-PCR detection. J Mol Med (Berl). 2018; 96:85-96. https://doi.org/10.1007/s00109-017-1600-y PMID:29098316

25. Tarnavski O. Mouse surgical models in cardiovascular research. Methods Mol Biol. 2009; 573:115-37. https://doi.org/10.1007/978-1-60761-247-6 7 PMID: 19763925

26. Jain A, Ravi V, Muhamed J, Chatterjee K, Sundaresan NR. A simplified protocol for culture of murine neonatal cardiomyocytes on nanoscale keratin coated surfaces. Int J Cardiol. 2017; 232:160-70. https://doi.org/10.1016/j.ijcard.2017.01.036

PMID:28096043

27. Li X, Liu CX, Xue W, Zhang Y, Jiang S, Yin QF, Wei J, Yao RW, Yang L, Chen LL. Coordinated circRNA Biogenesis and Function with NF90/NF110 in Viral Infection. Mol Cell. 2017; 67:214-27.e7. https://doi.org/10.1016/j.molcel.2017.05.023 PMID:28625552

28. Wang $\mathrm{H}$, He $\mathrm{P}$, Pan $\mathrm{H}$, Long J, Wang J, Li Z, Liu H, Jiang W, Zheng Z. Circular RNA circ-4099 is induced by TNF$\alpha$ and regulates ECM synthesis by blocking miR-616$5 p$ inhibition of Sox9 in intervertebral disc degeneration. Exp Mol Med. 2018; 50:1-14. https://doi.org/10.1038/s12276-018-0056-7 PMID:29651107 